\title{
Prevalence of Intestinal Parasitic Infections and Associated Risk Factors among the First-Cycle Primary Schoolchildren in Sasiga District, Southwest Ethiopia
}

\author{
Baye Sitotaw $\mathbb{D}^{1}$ and Wakgari Shiferaw ${ }^{2}$ \\ ${ }^{1}$ Department of Biology, Bahir Dar University, Ethiopia \\ ${ }^{2}$ ONRS, Sasiga District Education Office, Ethiopia \\ Correspondence should be addressed to Baye Sitotaw; mershabaye@gmail.com
}

Received 30 October 2019; Revised 15 February 2020; Accepted 28 February 2020; Published 13 March 2020

Academic Editor: José F. Silveira

Copyright ( $) 2020$ Baye Sitotaw and Wakgari Shiferaw. This is an open access article distributed under the Creative Commons Attribution License, which permits unrestricted use, distribution, and reproduction in any medium, provided the original work is properly cited.

\begin{abstract}
Intestinal parasitic infections (IPIs) have been major public health burdens in low-income countries like Ethiopia. Studies in different areas of Ethiopia have shown a high prevalence of IPIs in poor families. A similar study has not been conducted in Sasiga District given that the area is possibly at high-risk of IPIs due to the prevailing risk factors. This study is aimed at assessing the prevalence of IPIs and associated risk factors among schoolchildren in Sasiga District, southwest Ethiopia. A school-based cross-sectional study was conducted from December 2018 to March 2019 to estimate the prevalence of IPIs and associated risk factors among the study participants. A total of 383 children were selected using resident-type and grade-level stratified systematic random sampling technique. Stool samples were examined microscopically using direct wet mount and formal-ether concentration techniques. A structured questionnaire was used to get information on the associated risk factors. Data were analyzed using SPSS version 20 and $p$ value of $\leq 0.05$ was taken as statistically significant. The overall prevalence of IPIs among the children was $62.4 \%$ (239/383). Single, double, and triple infections were $49.9 \%, 10.7 \%$, and $1.83 \%$, respectively. Residence, family income, place of defecation, source of drinking water, shoe-wearing habit, handwashing habit after toilet use, ways of waste disposal, and cleanliness of fingernail were the most important predictors of IPIs $(p<0.05)$. Ascaris lumbricoides (22.7\% (87/383)) and hookworms (20.6\% (79/383)) were the most prevalent parasites, followed by Entamoeba histolytica (8.1\%), Trichuris trichiura (7.6\%), Giardia intestinalis (6.5\%), Hymenolepis nana (5.7\%), and Schistosoma mansoni (4.4\%), in that order. Sasiga District primary schoolchildren are likely at a high burden of IPIs. Intensive health education on personal hygiene and environmental sanitation is needed.
\end{abstract}

\section{Introduction}

Intestinal parasitic infections (IPIs) have been a big concern for low-income countries as they are the major cause of high morbidity and mortality. Most infectious diseases caused by members of the intestinal parasites (protozoan and helminths) have been considered as Neglected Tropical Diseases (NTDs) [1-4] and are affecting a large section of poor communities. Intestinal helminthiasis and protozoan infections are widespread throughout the world [5-7], and in particular, millions of people in low-income countries are infected and/or ill with parasitic infections (PIs). Due to this, ending epidemics of NTDS through the control of the transmission of IPIs and the mitigation of possible risk factors is one of the sustainable development goals (SDG) of the United Nations (2030 Agenda; Goal 3.3).

Regardless of huge budget mobilization on health improvement following SDG and significant improvements on the diagnosis of parasitic diseases and subsequent treatments $[8,9]$, any reduction has not shown, and IPIs happen to be the major public health problem in low-income countries primarily affecting schoolchildren [10-25]. Factors 
related to poverty, lack of awareness, and unavailability of sufficient health care as well as the prevailing bad climatic and environmental conditions are the most aggravating risk factors for IPIs.

Children are affected by IPIs far more than adults due to their higher nutritional requirements and less developed immune systems. In children, IPIs affect growth rate, protein-energy balance, and iron availability and consequently reduce mental development [1, 26, 27]. Globally, millions of preschoolers and schoolchildren are vulnerable to infections by parasitic worms and pathogenic protozoan species $[1,3,4]$ and are demanding urgent treatment and preventive interventions.

The protozoan parasite (Entamoeba histolytica and Giardia intestinalis/lamblia) and soil-transmitted helminths (Ascaris lumbricoides, Trichuris trichiura, and hookworm) are the most prevalent intestinal parasites causing high morbidity and mortality in sub-Saharan Africa, affecting nearly all inhabitants at some point in their lives $[1,10,13,15$, 18-25]. The prevalence of IPIs in the region is reported to be as high as $84 \%$ in Ethiopia [20], 90\% in Central Sudan [28], and $84.7 \%$ in Burkina Faso [29].

To accelerate the country's progress in meeting some of the Millennium Development Goals, the Federal Ministry of Health of Ethiopia started the health extension program since 2004. The health extension workers have been trained and assigned at almost every village in rural as well as urban areas. Despite the efforts, Ethiopia is still at a high burden of IPIs due to the aforementioned sociodemographic variables, behavioral factors, personal hygiene, and environmental sanitation factors $[1,18-26,29-32]$. Particularly, ascariasis, hookworm, and trichuriasis are listed among the most common public health burdens in Ethiopia [3]. Studies in the different regions of Ethiopia have shown a considerably high prevalence of IPIs. For instance, an extremely high prevalence $(84 \%)$ was reported among Debre Elias primary school children (northwest Ethiopia) [20]. In other studies conducted in the different regions of Ethiopia, the overall prevalence of IPIs, ranging from $54.5 \%$ to $83 \%$, was reported from primary schoolchildren $[18,19,21-25,30-32]$. To get a deeper insight into the magnitude of the problem and design effective intervention mechanisms, more information is needed from different localities where similar studies have not been conducted.

Clinical and health center reports (from years 2015 to 2017) in Sasiga District indicated that IPI was the main reason why many people visit health facilities. However, there was no scientific study conducted on the prevalence of IPIs and associated risk factors among schoolchildren in the study area. Therefore, the objective of this study was to estimate the prevalence of IPIs and associated risk factors among the first-cycle primary schoolchildren in Sasiga District, southwest Ethiopia.

\section{Methods}

2.1. Study Design and Study Area. A school-based crosssectional study was conducted from December 2018 to March 2019 to determine the prevalence of IPIs and the asso- ciated risk factors among the first-cycle primary schoolchildren in Sasiga District, southwest Ethiopia. Sasiga District is located $346 \mathrm{~km}$ southwest of Addis Ababa (the capital city of Ethiopia). The district is located at the geographical location of $9^{\circ} 10^{\prime} \mathrm{N}$ and $36^{\circ} 30^{\prime} \mathrm{E}$ and elevations of about 1,742 to 2,034 meters above sea level. Sixty percent of the district is midland to highland while $40 \%$ is a lowland agroecological zone. The annual mean temperature in the district ranges from $25^{\circ} \mathrm{C}$ to $32^{\circ} \mathrm{C}$ while the annual rainfall ranges from $1,200 \mathrm{~mm}$ to $1,800 \mathrm{~mm}$.

Based on the 2015 national housing and population census, the total population of the district was 105,603 (53,990 males and 51,613 females) (Sasiga District Administration Office, 2018). In Sasiga District, there are 6 governmental health centers, 14 private clinics, and 32 community health posts with a total of 187 health workers. In this district, access to clean water was low; as a result, people were forced to use various unprotected water sources such as rivers (authors' observation).

2.2. Study Population and Sample Size Determination. Based on the information from Sasiga District Educational Office (2018), there were 12 first-cycle (grade 1 to 4 ) primary schools, 30 first and second-cycle (grade 1 to 8 ) primary schools, and 7 secondary schools in the district. The study population was all schoolchildren enrolled in the first-cycle primary schools in Sasiga District (from grades 1 to 4). And the study participants were all students who are volunteers (gave consent) to participate in the study and those who did not take any antiintestinal parasitic drugs within 2 weeks. Of the 12 first-cycle primary schools in the district, 4 schools (2 from urban and 2 from rural) were selected for this study. There were 878 students enrolled in these 4 schools in the 2018/2019 academic year. Since there was no similar study previously conducted in the area, a $50 \%$ prevalence rate of IPIs was taken assuming that IPIs are significantly prevalent among students in the firstcycle primary schools in Sasiga District. Accordingly, the minimum number of sample size $(n)$ required was determined using a single population proportion formula for cross-sectional surveys [21], i.e., $n=Z^{2} \mathrm{p}(1-\mathrm{p}) / d^{2}=$ $1.96^{2} \times 0.50 \times 0.50 / .05^{2}=384$ students. To compensate for the nonrespondents and to minimize errors probably arising from the likelihood of noncompliance, $10 \%$ was added giving a final sample size of 422 study participants.

Study participants were selected from the students stratified into four schools using a quota system. Again, quota system was used to select students from the four grade levels at each school. The actual number of students participated in the study from each grade level was selected by systematic random sampling technique to include 422 schoolchildren.

2.3. Sample Collection and Processing. A structured questionnaire based on known risk factors was developed in English and translated into Oromo language (local language). The participants of the study or parents in the case of younger children were interviewed to obtain information on sociodemographic characteristics, behavioral factors, hygienic practices, and environmental sanitation factors (see Table 1 and 
TABLE 1: Sociodemographic characteristics of the study participants.

\begin{tabular}{|c|c|c|c|}
\hline Sociodemographic variables & Categories & Frequency & Percentage \\
\hline \multirow{4}{*}{ Grade level } & 1 & 120 & 31.3 \\
\hline & 2 & 112 & 29.2 \\
\hline & 3 & 83 & 21.7 \\
\hline & 4 & 68 & 17.8 \\
\hline \multirow{3}{*}{ Age groups (years) } & Childhood $(<5)$ & 8 & 2.1 \\
\hline & Mid childhood (6-11) & 320 & 83.5 \\
\hline & Early adolescent (12-18) & 55 & 14.4 \\
\hline \multirow{2}{*}{ Sex } & Male & 187 & 48.8 \\
\hline & Female & 196 & 51.2 \\
\hline \multirow{2}{*}{ Residence } & Urban & 201 & 52.5 \\
\hline & Rural & 182 & 47.5 \\
\hline \multirow{3}{*}{ Family size } & $<4$ & 45 & 11.75 \\
\hline & $5-7$ & 191 & 49.9 \\
\hline & $8-10$ & 147 & 38.4 \\
\hline \multirow{3}{*}{ Family monthly income (ETB)* } & $<800$ & 163 & 42.6 \\
\hline & $800-2,000$ & 170 & 44.9 \\
\hline & $>2,000$ & 50 & 13.1 \\
\hline \multirow{2}{*}{ Father education } & Primary school & 221 & 57.7 \\
\hline & Secondary school and above & 162 & 42.3 \\
\hline \multirow{2}{*}{ Mother education } & Primary school & 347 & 90.6 \\
\hline & Secondary school and above & 36 & 9.44 \\
\hline \multirow{4}{*}{ Father occupation } & Farmer & 332 & 86.7 \\
\hline & Government employee & 16 & 4.2 \\
\hline & Merchant & 20 & 5.2 \\
\hline & Daily laborer & 15 & 3.9 \\
\hline \multirow{4}{*}{ Mother occupation } & Housewife & 350 & 91.4 \\
\hline & Government employee & 9 & 1.3 \\
\hline & Merchant & 21 & 5.5 \\
\hline & Daily laborer & 3 & 1.6 \\
\hline
\end{tabular}

${ }^{*} 1$ USD is about 30 ETB.

additional file 1 for the details). Then, the responses were translated back into English. The questionnaire was pretested using forty individuals outside the study area in a nonstudy sample population.

For parasitological analysis, fresh stool samples were collected from each schoolchild. The children were instructed properly and were given clean labeled collection cups along with applicator sticks, and from each student, about $2 \mathrm{~g}$ of fresh stool was collected. At the time of collection, date of sampling, school name, the name of the participant, age, and sex were recorded for each subject in a recording format. Stool samples were preserved in a 10\% formalin before transported to the health center laboratory. A portion of each of the stool samples was processed and examined microscopically using direct wet mount and formal-ether concentration techniques following the procedures in WHO guidelines [33]. All developmental stages of the parasites (cyst, egg, larvae, and adult) were recorded.
2.4. Limitations of the Study. The study was limited to only students in the first-cycle of primary school. Including second-cycle primary schools would have been better to get a bigger picture of the prevalence of IPIs among schoolchildren of the area. The study was also limited to the presence or absence of infections without quantifying the parasite load, which may not show that the infected students were diseased. Moreover, a self-reported data collection method was used which may also bias the information.

2.5. Data Analysis. Statistical Package for Social Science (SPSS) software version 20 was used to analyze the collected data. Chi-square $\left(\chi^{2}\right)$ test was performed to verify the possible association between the prevalence of IPIs and sociodemographic characteristics, behavioral factors, hygienic practices, and environmental sanitation factors. Logistic regression was used to measure the strengths of association between the prevalence of infection and the risk factors using odds ratio. 
In the modeling process, a univariate analysis (crude odds ration) was first done with a 0.25 level of significance to select the candidate variables for multivariate analysis (adjusted odds ratio). The variables, significant at the univariate analysis, were then included in the multivariate analysis [34]. Values were considered significant at $p \leq 0.05$.

\section{Results}

3.1. Sociodemographic Characteristics of the Study Participants. From a total of 422 students selected for these study, 39 (10.2\%) were excluded due to either incomplete information or insufficient fecal specimen production. As a result, 383 $(90.76 \%)$ gave stool for intestinal parasitic examination and filled questionnaires on associated risk factors. From 383 study participants, about half were urban dwellers; female participants were slightly greater $(51.2 \%)$ than males (48.8\%); most were from farmer father (87\%) and housewife mother (91\%), and most were from families who earned Birr 800-2,000 monthly (Table 1). The age of the participants ranged from 5 to 15 years, and most of the participants $(83.3 \%)$ were 6-11 years old. Similarly, most of the participants were from mothers who attended only primary school (90.6\%) and a little more than half (57.7\%) from fathers who attended only primary school. Furthermore, most of the participants (91\%) came from family sizes of 5 and above (Table 1).

3.2. Prevalence of Intestinal Parasitic Infections among the Study Participants. Three hundred eighty-three students were examined for IPIs, and $62.40 \%$ of these participants were positive for at least one intestinal parasite. The rates of single, double, and triple infections were 191 (49.9\%), 41 $(10.7 \%)$, and $7(1.83 \%)$, respectively. The prevalences of protozoa, helminths, and mixed infections were $26(6.8 \%)$, $181(47.26 \%)$, and $32(8.36 \%)$, respectively. Identified intestinal parasites, in order of decreasing prevalence rate, were Ascaris lumbricoides $(22.7 \%$ (87/383)) and Hookworms (20.6\% (79/383)), Entamoeba histolytica (8.1\%), Trichuris trichiura (7.6\%), Giardia intestinalis (6.5\%), Hymenolepis nana (5.7\%), and Schistosoma mansoni (4.4\%) (Table 2).

\subsection{Association of the Different Risk Factors with Intestinal} Parasitic Infections. The overall infection rate was similar in males (62.6\%) and females (62.2\%). Moreover, there was no significant variation in the infection rate of IPIs among the students in the different age groups, grade level, and family size $(p>0.05)$. However, there was a statistically significant association between IPIs and some other sociodemographic factors including residence, family income, educational status, and occupation of the parents. Accordingly, a significantly higher prevalence rate of IPIs was observed among students living in rural (76.4\%) than urban (49.8\%) areas. Similarly, students from families who earned less than Birr 800 (Ethiopian currency) were more infected with IPs (86.5\%) compared with families who earned more than Birr $800(44 \%)(p<0.05)$. Likewise, students from families who attended only primary school were more infected (64.5$71.1 \%$ ) with IPs compared with those from families who attended secondary school and above (41.7-50.6\%). Types of mother and father occupation had also a significant effect on the infection rate of IPIs (Supplementary file 1).

Except for the habits of eating raw meat, all other factors related to the practices in personal hygiene and environmental sanitation had significant effects on the rate of IPIs among the students (Supplementary file 1). The prevalence of IPIs among the participants who had varying habits (did always, sometimes, or not all) of shoe wearing, hand washing after toilet use, and eating unwashed fruits and vegetables was statistically significant $(p<0.002)$. Similarly, cleanliness of students' fingernails (clean or unclean), place of defecation (open-field or in latrine), source of drinking water (river, well, or pipe), and ways of waste disposal (open dump, burry, or burn) had significant effects on the prevalence rate of IPIs among the participants $(p<0.002)$. Consequently, high prevalence rates of IPIs were observed in children who did not wear shoe at all and wore sometimes, who did not wash their hands always after toilet use, who had habits of open-field defecation, who ate unwashed fruits and vegetables, and who had dirty fingernails (close observation by the investigator) (Supplementary file 1).

3.4. Logistic Regression Analysis (LRA) of the Most Important Risk Factors for IPIs. The most important risk factors for IPIs among the first-cycle primary schoolchildren in Sasiga District were identified using Multivariable Logistic Regression Analyses (MLRA) (Table 3). In the modeling process, a univariate analysis was first done with a 0.25 level of significance to select the candidate variables for multivariable analysis. Sixteen (out of eighteen) variables, significant at the univariate analysis, were included in the multivariable analysis [34]. Residence, family income, father occupation, handwashing habit after toilet use, place of defecation, source of drinking water, habit of wearing shoes, ways of waste disposal, and unclean fingernails were significantly associated $(p<0.05)$ with IPIs (Table 3$)$ and were found to be the most important predictors of IPIs among the students in the firstcycle primary school in Sasiga District.

Accordingly, the likelihood of being infected by intestinal parasites (IPs) was increased by fivefold ( $\mathrm{AOR}=5.45, \mathrm{CI}=$ $2.293-12.949 ; p \leq 0.001)$ in students who lived in rural area than in urban area (Table 3 ); the risk of being infected by IPs was increased by 4 times $(\mathrm{AOR}=4.159 ; \mathrm{CI}=1.257-13.764$; $p=0.02$ ) in students whose parents' monthly income was less than Birr 800 compared to students whose parents' income was more than Birr 2,000; the likelihood of being infected by IPs was increased eightfold $(\mathrm{AOR}=8.377$; $\mathrm{CI}=3.552-$ $19.752 ; p \leq 0.001)$ and 12 -fold $(\mathrm{AOR}=12.127 ; \mathrm{CI}=4.247-$ 34.626; $p \leq 0.001)$ in students who buried wastes and dumped wastes in open field, respectively, than students who burned the wastes; the risk of being infected by IPs was increased by almost fivefold in students who defecated in open field $(\mathrm{AOR}=4.747 ; \mathrm{CI}=1.820-12.379 ; p=0.001)$ than those who used latrine; students who did not wear shoes were about 7 times $(\mathrm{AOR}=6.889$; $\mathrm{CI}=3.518-$ 13.489; $p \leq 0.001$ ) more likely to be infected with IPs than those who always wore shoes; students who did not regularly wash their hands after toilet use were about 2 times 
TABLE 2: Association of individual intestinal parasites with sociodemographic variables.

\begin{tabular}{|c|c|c|c|c|c|c|c|}
\hline Intestinal parasites & 1 & 2 & 3 & 4 & Total (\%) & $\chi^{2}$ & $p$ value \\
\hline \multicolumn{8}{|c|}{ Grade level no. (\%) } \\
\hline A. lumbricoides & $32(26.7)$ & $21(18.8)$ & $18(21.7)$ & $16(23.5)$ & $87(22.7)$ & 2.146 & 0.543 \\
\hline Hookworms & $28(23.3)$ & $25(22.3)$ & $11(13.3)$ & $15(22.1)$ & $79(20.6)$ & $3.575^{\mathrm{a}}$ & 0.311 \\
\hline E. histolytica & $11(9.2)$ & $10(8.9)$ & $5(6)$ & $5(7.4)$ & $31(8.1)$ & 0.819 & 0.845 \\
\hline T. trichiura & $10(8.3)$ & $6(5.4)$ & $8(9.6)$ & $5(7.4)$ & $29(7.6)$ & 1.396 & 0.707 \\
\hline G. intestinalis & $10(8.3)$ & $12(10.7)$ & $1(1.2)$ & $2(2.9)$ & $25(6.5)$ & 9.147 & $0.027^{*}$ \\
\hline H. nana & $5(4.2)$ & $10(8.9)$ & $4(4.8)$ & $3(4.4)$ & $22(5.7)$ & 3.003 & 0.391 \\
\hline S. mansoni & $2(1.7)$ & $6(5.4)$ & $4(4.8)$ & $5(7.4)$ & $17(4.4)$ & 3.787 & 0.285 \\
\hline \multicolumn{8}{|c|}{ Age in years no. (\%) } \\
\hline & $\leq 5(\%)$ & 6-11 (\%). & $12-18(\%)$ & & & & \\
\hline A. lumbricoides & $4(50)$ & $65(20.3)$ & $18(32.7)$ & - & $87(22.7)$ & 7.585 & $0.023^{*}$ \\
\hline Hookworms & $2(25)$ & $67(20.9)$ & $10(18.2)$ & - & $79(20.6)$ & 0.313 & 0.855 \\
\hline E. histolytica & $1(12.5)$ & $29(9.1)$ & $1(1.8)$ & - & $31(8.1)$ & 3.524 & 0.172 \\
\hline T. trichiura & - & $25(6.5)$ & $4(7.4)$ & - & $29(7.6)$ & 0.689 & 0.709 \\
\hline G. intestinalis & $1(12.5)$ & $22(6.9)$ & $2(3.6)$ & - & $25(6.5)$ & 1.285 & 0.526 \\
\hline H. nana & - & $20(6.2)$ & $2(3.6)$ & - & $22(5.7)$ & $1.090)$ & 0.58 \\
\hline S. mansoni & - & $11(3.4)$ & $6(10.9)$ & - & $17(4.4)$ & 6.556 & $0.038^{*}$ \\
\hline \multicolumn{8}{|c|}{ Sex no. $(\%)$} \\
\hline & Male & Female & & & & & \\
\hline A. lumbricoides & $41(21.9)$ & $46(23.5)$ & - & - & $87(22.7)$ & 0.13 & 0.718 \\
\hline Hookworms & $37(19.8)$ & $42(21.4)$ & - & - & $79(20.6)$ & 0.158 & 0.691 \\
\hline E. histolytica & $13(7.0)$ & $18(9.2)$ & - & - & $31(8.1)$ & 0.641 & 0.423 \\
\hline T. trichiura & $17(9.1)$ & $12(6.1)$ & - & - & $29(6.7)$ & 1.205) & 0.272 \\
\hline G. intestinalis & $11(8.9)$ & $14(7.1)$ & - & - & $25(6.5)$ & 0.249 & 0.618 \\
\hline H. nana & $14(7.5)$ & $8(4.1)$ & - & - & $22(5.7)$ & 2.049 & 0.152 \\
\hline S. mansoni & $7(3.7)$ & $10(5.1)$ & - & - & $17(4.4)$ & 0.417 & 0.519 \\
\hline \multicolumn{8}{|c|}{ Residence no. (\%) } \\
\hline & Urban (\%) & Rural (\%) & & & & & \\
\hline A. lumbricoides & $41(20.4)$ & $46(25.3)$ & & & $87(22.7)$ & 1.294 & 0.255 \\
\hline Hookworms & $41(20.4)$ & $38(20.9)$ & & & $79(20.6)$ & 0.014 & 0.907 \\
\hline E. histolytica & $20(10)$ & $11(6)$ & & & $31(8.1)$ & 1.959 & 0.162 \\
\hline T. trichiura & $6(3)$ & $23(12.6)$ & & & $29(7.6)$ & 12.72 & $0.001^{* *}$ \\
\hline G. intestinalis & $10(5)$ & $15(8.2)$ & & & $25(6.5)$ & 1.67 & 0.196 \\
\hline H. nana & 0 & $22(12.1)$ & & & $22(5.7)$ & 25.78 & $0.001^{* *}$ \\
\hline \multirow[t]{3}{*}{ S. mansoni } & $10(5)$ & $7(3.8)$ & & & $17(4.4)$ & 0.278 & 0.592 \\
\hline & \multicolumn{3}{|c|}{ Family size no. (\%) } & & & & \\
\hline & $2-4$ & $5-7$ & $8-10$ & & & & \\
\hline A. lumbricoides & $12(26.7)$ & $51(26.7)$ & $24(16.3)$ & - & $87(22.7)$ & 5.547 & 0.062 \\
\hline Hookworms & $5(11.1)$ & $43(22.5)$ & $31(21.1)$ & - & $79(20.6)$ & 2.923 & 0.232 \\
\hline E. histolytica & 1 & $24(12.6)$ & $6(4.1)$ & - & $31(8.1)$ & 2.106 & 0.147 \\
\hline T. trichiura & $4(8.9)$ & $12(6.3)$ & $13(8.8)$ & - & $29(7.6)$ & 0.905 & 0.636 \\
\hline G. intestinalis & 1 & $12(6.3)$ & $12(8.2)$ & - & $25(6.5)$ & 2.030 & 0.362 \\
\hline H. nana & $3(6.7)$ & $13(6.8)$ & $6(4.1)$ & - & $22(5.7)$ & 1.219 & 0.544 \\
\hline S. mansoni & $2(4.4)$ & $7(3.7)$ & $8(5.4)$ & & $17(4.4)$ & 0.346 & 0.556 \\
\hline
\end{tabular}


TABle 2: Continued.

\begin{tabular}{|c|c|c|c|c|c|c|c|}
\hline Intestinal parasites & 1 & 2 & 3 & 4 & Total $(\%)$ & $\chi^{2}$ & $p$ value \\
\hline & \multicolumn{7}{|c|}{ Family monthly income (ETB) no. (\%) } \\
\hline & $\leq 800(\%)$ & $800-2,000(\%)$ & $\geq 2,000(\%)$ & & & & \\
\hline A. lumbricoides & $57(35)$ & $23(13.5)$ & $7(14)$ & & $87(22.7)$ & 24.28 & $0.001^{* *}$ \\
\hline Hookworms & $41(25)$ & $31(18)$ & $7(14)$ & & $79(20.6)$ & 3.975 & 0.137 \\
\hline E. histolytica & $21(12.9)$ & $8(4.7)$ & $2(4)$ & & $31(8.1)$ & 8.776 & $0.012^{*}$ \\
\hline T. trichiura & $19(11.7)$ & $9(5.3)$ & $1(2)$ & & $29(7.6)$ & 7.364 & $0.025^{*}$ \\
\hline G. intestinalis & $13(8)$ & $8(4.7)$ & $4(8)$ & & $25(6.5)$ & 1.662 & 0.436 \\
\hline H. nana & $14(8)$ & $6(3.5)$ & $2(4)$ & & $22(5.7)$ & 4.258 & 0.119 \\
\hline \multirow[t]{3}{*}{ S. mansoni } & $9(2.3)$ & $5(1.3)$ & $3(0.8)$ & & $17(4.4)$ & 1.637 & 0.441 \\
\hline & & Father education no. (\%) & & & & & \\
\hline & Primary school & Secondary school and above & & & & & \\
\hline A. lumbricoides & $60(27.1)$ & $27(17)$ & - & & $87(22.7)$ & 7.009 & $0.03^{*}$ \\
\hline Hookworms & $54(24.4)$ & $25(16)$ & - & & $79(20.6)$ & 5.399 & 0.067 \\
\hline E. histolytica & $25(11.3)$ & $6(3.9)$ & - & & $31(8.1)$ & 7.409 & $0.025^{*}$ \\
\hline T. trichiura & $17(7.7)$ & $12(7.4)$ & - & & $29(7.6)$ & 0.505 & 0.777 \\
\hline G. intestinalis & $12(5.4)$ & $13(8)$ & - & & $25(6.1)$ & 1.502 & 0.472 \\
\hline H. nana & $12(5.4)$ & $10(6.5)$ & - & & $22(5.7)$ & 0.61 & 0.737 \\
\hline \multirow[t]{3}{*}{ S. mansoni } & $13(5.9)$ & $4(2.6)$ & - & & $17(4.4)$ & 2.673 & 0.263 \\
\hline & & Mother education no. (\%) & & & & & \\
\hline & Primary school & Secondary school and above & & & & & \\
\hline A. lumbricoides & $80(23.1)$ & $7(21.9)$ & - & & $87(22.7)$ & 1.211 & 0.546 \\
\hline Hookworms & $76(19.8)$ & $3(9.4)$ & - & & $79(20.6)$ & 3.859 & 0.145 \\
\hline E. histolytica & $30(8.6)$ & $1(3.1)$ & - & & $31(8.1)$ & $1.556)$ & 0.459 \\
\hline T. trichiura & $24(6.9)$ & $1(3.1)$ & - & & $25(6.5)$ & 0.973 & 0.615 \\
\hline G. intestinalis & $21(6.1)$ & $1(3.1)$ & - & & $22(5.7)$ & 0.71 & 0.701 \\
\hline H. nana & $17(4.9)$ & $0(0.00)$ & - & & $17(4.4)$ & 1.211 & 0.807 \\
\hline \multirow[t]{3}{*}{ S. mansoni } & $27(7)$ & $2(0.5)$ & - & & $29(7.6)$ & 0.429 & 0.807 \\
\hline & & Father occupation no. (\%) & & & & & \\
\hline & Daily laborer & Farmer & Govt. employee & Merchant & & & \\
\hline A. lumbricoides & $9(60)$ & $73(22)$ & $1(6.2)$ & $4(20)$ & $87(22.7)$ & 14.53 & $0.002^{*}$ \\
\hline Hookworms & $5(33.3)$ & $67(20.2)$ & $3(18.8)$ & $4(20)$ & $79(20.6)$ & 1.559 & 0.669 \\
\hline E. histolytica & $3(20)$ & $27(8.1)$ & - & $1(6.7)$ & 31 & 4.525 & 0.21 \\
\hline T. trichiura & $1(6.7)$ & $21(6.3)$ & $1(6.2)$ & $2(10)$ & $25(6.5)$ & 0.42 & 0.936 \\
\hline G. intestinalis & $1(6.7)$ & $20(6)$ & - & $1(5)$ & $22(5.7)$ & 1.067 & 0.785 \\
\hline H. nana & - & $16(4.8)$ & - & $1(5)$ & $17(4.4)$ & 1.586 & 0.667 \\
\hline \multirow[t]{3}{*}{ S. mansoni } & $5(33.5)$ & $23(6.9)$ & $1(6.2)$ & - & $29(7.6)$ & 16.1 & $0.001^{* *}$ \\
\hline & & Mother occupation no. (\%) & & & & & \\
\hline & Daily laborer & Housewife & Govt. employee & Merchant & & & \\
\hline A. lumbricoides & $1(33.3)$ & $82(23.4)$ & - & $4(19)$ & $87(22.7)$ & 3.1 & 0.376 \\
\hline Hookworms & $1(33.3)$ & $76(21.7)$ & - & $2(9.5)$ & $79(20.6)$ & 4.469 & 0.215 \\
\hline E. histolytica & $2(66.7)$ & $27(7.7)$ & - & $2(0.5)$ & $31(8.1)$ & 14.75 & $0.002^{*}$ \\
\hline T. trichiura & - & $25(7.1)$ & - & - & $25(6.5)$ & 2.522 & 0.471 \\
\hline G. intestinalis & $1(33.3)$ & $20(5.7)$ & - & $1(4.8)$ & $22(5.7)$ & 4.804 & 0.187 \\
\hline H. nana & - & $17(4.9)$ & - & - & $17(4.4)$ & 1.677 & 0.642 \\
\hline S. mansoni & - & $28(8)$ & - & $1(4.8)$ & $29(7.6)$ & 1.312 & 0.721 \\
\hline Single infection & - & - & - & - & $191(49.9)$ & - & - \\
\hline Double infection & - & - & - & - & $41(10.7)$ & - & - \\
\hline Triple infection & - & - & - & - & $7(1.8)$ & - & - \\
\hline Overall infection & - & - & - & - & $239(62.4)$ & & \\
\hline
\end{tabular}

${ }^{* *}$ Statistically significant at $p \leq 0.001$. ${ }^{*}$ Statistically significant at $p<0.05$. 
TABLE 3: Risk factors associated with overall intestinal parasitic infections.

\begin{tabular}{|c|c|c|c|c|c|c|}
\hline \multicolumn{7}{|c|}{ Sociodemographic variables } \\
\hline \multicolumn{7}{|c|}{ Number and percentage of parasite-infected student } \\
\hline Variables & Categories & $\begin{array}{l}\text { Total no. } \\
\quad(\%)\end{array}$ & $\begin{array}{l}\text { Positive no. } \\
\text { (\%) }\end{array}$ & $\begin{array}{l}\text { Negative no. } \\
(\%)\end{array}$ & $\operatorname{AOR}(95 \% \mathrm{CI})$ & $p$ value \\
\hline \multirow{4}{*}{ Grade level } & 1 & $120(31.3)$ & $80(66.6)$ & $40(33.33)$ & $0.878(0.28-2.6)$ & 0.817 \\
\hline & 2 & $112(29.2)$ & $71(63.4)$ & $41(36.6)$ & $1.379(0.45-4.2)$ & 0.571 \\
\hline & 3 & $83(21.7)$ & $44(53)$ & $39(47)$ & $0.961(0.28-3.2)$ & 0.949 \\
\hline & 4 & $68(17.8)$ & $44(64.7)$ & $24(35.3)$ & 1 & \\
\hline \multirow{2}{*}{ Residence } & Rural & $182(47.5)$ & $139(76.4)$ & $43(23.6)$ & $5.41(2.3-12.9)$ & $0.000^{* *}$ \\
\hline & Urban & $201(52.5)$ & $100(49.8)$ & $101(50.2)$ & 1 & \\
\hline \multirow{3}{*}{ Family size } & $2-4$ & $45(11.75)$ & $23(51.1)$ & $22(48.9)$ & $1.865(0.5-6.31)$ & 0.316 \\
\hline & $5-7$ & $191(49.9)$ & $129(67.5)$ & $62(32.5)$ & $1.389(0.61-3.1)$ & 0.429 \\
\hline & $8-10$ & $\begin{array}{c}147 \\
(38.38)\end{array}$ & $87(59.2)$ & $60(40.8)$ & 1 & \\
\hline \multirow{3}{*}{ Family monthly income (ETB) } & $<800$ & $163(42.6)$ & $141(86.5)$ & $22(13.5)$ & $4.159(1.25-13.76)$ & $0.02^{*}$ \\
\hline & $800-2,000$ & $170(44.9)$ & $76(44.7)$ & $94(55.3)$ & $1.383(0.4-4.17)$ & 0.585 \\
\hline & $>2.000$ & $50(13.1)$ & $22(44)$ & $28(56)$ & 1 & \\
\hline \multirow[b]{2}{*}{ Father education } & Primary school & $221(57.7)$ & $157(71.1)$ & $64(28.9)$ & $1.229(0.5-2.64)$ & 0.598 \\
\hline & $\begin{array}{l}\text { Secondary school and } \\
\text { above }\end{array}$ & $162(42.3)$ & $82(50.6)$ & $80(49.4)$ & 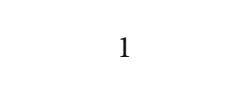 & \\
\hline \multirow[b]{2}{*}{ Mother education } & Primary school & $347(90.6)$ & $224(64.5)$ & $123(35.5)$ & $0.990(0.22-4.35)$ & 0.990 \\
\hline & $\begin{array}{l}\text { Secondary school and } \\
\text { above }\end{array}$ & $36(9.44)$ & $15(41.7)$ & $21(58.3)$ & 1 & \\
\hline \multirow{2}{*}{ Fathers' occupation } & Farmer & $332(86.7)$ & $208(62.6)$ & $124(37.4)$ & $0.624(0.19-2.05)$ & 0.438 \\
\hline & Others & $51(13.3)$ & $31(60.78)$ & $20(21.21)$ & 1 & \\
\hline \multirow{2}{*}{ Mothers' occupation } & Housewife & $350(91.4)$ & $229(65.4)$ & $121(34.6)$ & $1.88(0.368-9.67)$ & 0.447 \\
\hline & Others & $33(8.6)$ & $10(30.3)$ & $23(67.7)$ & 1 & \\
\hline \multicolumn{7}{|c|}{ Behavioral factors, hygienic practice, and environmental variables } \\
\hline \multirow{3}{*}{ Place of defecation } & Open field & $148(38.6)$ & $139(93.9)$ & $9(6.1)$ & $4.747(1.82-12.37)$ & $0.001^{* *}$ \\
\hline & Latrine & $235(59.8)$ & $100(42.5)$ & $135(47.5)$ & 1 & \\
\hline & River & $149(38.9)$ & $125(83.9)$ & $24(16.1)$ & $3.124(1.325-7.36)$ & $0.009^{*}$ \\
\hline \multirow[t]{2}{*}{ Source of drinking water } & Well & $27(7)$ & $22(81.5)$ & $5(18.5)$ & $2.054(0.47-8.95)$ & 0.338 \\
\hline & Tap & $207(54)$ & $92(44.4)$ & $115(55.6)$ & 1 & \\
\hline \multirow{3}{*}{ Shoe-wearing habit } & Always & $272(66)$ & $143(52.6)$ & $129(47.4)$ & 1 & \\
\hline & Sometimes & $95(24.8)$ & $84(88.4)$ & $11(11.6)$ & $6.889(3.51-13.48)$ & $0.000^{* *}$ \\
\hline & Not at all & $16(4.2)$ & $12(75)$ & $4(25)$ & $2.706(0.851-8.60)$ & .092 \\
\hline \multirow{3}{*}{$\begin{array}{l}\text { Habits of washing fruits and vegetables } \\
\text { before eating }\end{array}$} & Always & $150(21.4)$ & $74(49.3)$ & $76(50.7)$ & 1 & \\
\hline & Sometimes & $215(56.1)$ & $152(70.7)$ & $63(29.3)$ & $1.993(0.32-12.22)$ & 0.456 \\
\hline & Not at all & $18(4.7)$ & $15(83.3)$ & $3(16.7)$ & $1.77(0.77-4.04)$ & 0.174 \\
\hline \multirow{3}{*}{ Hand washing habit after toilet use } & Always & $163(42.6)$ & $73(44.8)$ & $90(55.2)$ & 1 & \\
\hline & Sometimes & $207(54)$ & $154(74.4)$ & $53(25.6)$ & $2.358(1.047-5.31)$ & $0.038^{*}$ \\
\hline & Not at all & $13(3.4)$ & $12(92.3)$ & $1(7.7)$ & $3.137(0.19-49.61)$ & 0.417 \\
\hline \multirow{3}{*}{ Raw meat-eating habit } & Frequent & $49(13)$ & $37(75.5)$ & $12(24.5)$ & $1.127(0.31-4.079)$ & 0.856 \\
\hline & Sometimes & $177(28.7)$ & $110(62.1)$ & $67(37.9)$ & $0.470(0.124-1.78)$ & 0.267 \\
\hline & Not at all & $157(41)$ & $94(59.9)$ & $63(40.1)$ & 1 & \\
\hline
\end{tabular}


TABLE 3: Continued.

\begin{tabular}{|c|c|c|c|c|c|c|}
\hline \multicolumn{7}{|c|}{ Sociodemographic variables } \\
\hline \multirow{3}{*}{ Ways of waste disposal } & Burning & $141(23.8)$ & $33(23.4)$ & $108(76.6)$ & 1 & \\
\hline & Burying & $91(23.8)$ & $78(85.7)$ & $13(14.3)$ & $12.12(4.24-34.62)$ & $0.000^{* *}$ \\
\hline & Open dump & $151(39.4)$ & $128(84.8)$ & $23(15.2)$ & $8.377(3.55-19.75)$ & $0.000^{* *}$ \\
\hline \multirow{2}{*}{ Fingernail cleanliness ${ }^{@}$} & Clean & $256(66.8)$ & $128(50)$ & $128(50)$ & 1 & \\
\hline & Not clean & $127(33.2)$ & $111(87.4)$ & $16(12.6)$ & $4.2(1.67-10.55)$ & $0.002^{*}$ \\
\hline
\end{tabular}

Note: $1=$ reference value; ${ }^{* *}$ statistically significant at $p \leq 0.001$; ${ }^{*}$ statistically significant at $p<0.05 ; \mathrm{AOR}=$ adjusted odds ratio; multivariate regression model for grade level, family size, residence, family monthly income, father education, mother education, father occupation, mother occupation, place of defecation, source of drinking water, shoe-wearing habit, habit of eating unwashed fruits and vegetables, handwashing habit after toilet use, raw meat-eating habits, ways of waste disposal, and fingernails cleanliness for intestinal parasitic infections; @ = finger nail cleanliness was evaluated by observing whether nails are trimmed or not.

$(\mathrm{AOR}=2.358 ; \mathrm{CI}=1.047-5.312 ; p=0.038)$ more likely to have IPs than those who washed their hands regularly after toilet use; students who used river as source of drinking water were 3 times $(\mathrm{AOR}=3.124 ; \mathrm{CI}=1.325-7.362$; $p=0.009$ ) more likely to have IPIs than those who used tap water for the same purpose; students who had unclean fingernails were more likely to have IPIs than those students who had clean fingernails (Table 3 ).

3.5. Risk Factors Associated with the Prevalence of Individual Parasites. Five helminths and two protozoan intestinal parasitic species were identified in this study, of which infections by Ascaris lumbricoides and Hookworm were the most prevalent (Table 2). Infections by the different parasites were associated with some particular groups of risk factors for each parasite (Table 2 and Supplementary file 2). In the MLRA model, residence, family size, and ways of waste disposal were predictors of A. lumbricoides infection. Accordingly, the risk of A. lumbricoides infection was increased by about threefold in students from the family size of less than $4 \quad(\mathrm{AOR}=2.706$; $\mathrm{CI}=1.043-$ 7.017; $p=0.041$ ) compared with those from the family size of 8 and above (Table 4). Similarly, the likelihood of being infected by $A$. lumbricoides was increased eightfold $(\mathrm{AOR}=8.033 ; \quad \mathrm{CI}=3.022-22.134 ; p \leq 0.001) \quad$ and fivefold $(\mathrm{AOR}=4.898 ; \mathrm{CI}=1.722-13.929 ; p=0.03) \quad$ in students who dumped wastes in open-field and buried wastes, respectively, than students who burned the wastes. Shoe-wearing habits and ways of waste disposal were predictors of hookworm infection among the study participants. Students who did not wear shoes were 8 times $(\mathrm{AOR}=8.346 ; \quad \mathrm{CI}=2.908-18.761 ; p=0.001)$ and who sometimes wore shoes were 3 times $(\mathrm{AOR}=3.648 ; \mathrm{CI}=$ $2.094-6.358 ; p=0.001)$ more likely to be infected with hookworm compared with students who always wore shoes. And students who buried wastes were about 3 times $(\mathrm{AOR}=2.97 ; \mathrm{CI}=1.29-6.838 ; p=0.011)$ more likely to be infected with hookworm compared to those who burned the wastes. As indicated in the Supplementary file 3, residence, raw meat-eating habits, and age were found to be predictors of infections by Trichuris trichiura, Hymenolepis nana, and Schistosoma mansoni, respectively.

\section{Discussion}

IPIs continue to challenge public health in low-income countries like Ethiopia. To identify high-risk communities and design effective intervention mechanisms, studies in different settings are a vital step. Based on this view, we estimated the prevalence of IPIs and identified associated risk factors among students attending the first-cycle primary schools in Sasiga District, southwest Ethiopia.

The overall prevalence of IPIs among the study participants was notably high $(62.4 \%)$, showing that this community is likely at a high burden of intestinal parasitosis given methodological limitations. Similarly, high-risk communities, especially school-aged children, have been shown through cross-sectional studies conducted in the different parts of Ethiopia [18, 20-25, 31, 32, 35-37]. Even, extreme prevalence rates (over $75 \%$ ) were reported from different regions of Ethiopia [18, 20, 21, 32]. The community-based accelerated expansion of health facilities in Ethiopia being operational since 2004 seems ineffective as a sound reduction in the prevalence of such neglected diseases was expected. Parts of communities in other low-income countries such as India (43-49\%) [12, 38], Nepal (52-59\%) [39, 40], Nigeria (58.3-81\%) [15, 41, 42], Burkina Faso (65$84.7 \%)[13,43]$, and Peru (100\%) [16] are also reported to be at high-risk of IPIs indicating that IPIs continue to be major threats to poor society. Low socioeconomic status, low educational level, and hence poor knowledge, attitude, and practices towards easily preventable disease, poor personal hygiene, and environmental sanitation, lack of potable, and sufficient drinking water are the most important risk factors for the high infection rate of intestinal parasites among poor communities.

In this study, the infection rate was strongly associated $(p \leq 0.009)$ with 13 out of 18 risk factors considered (Supplementary file 1). All these factors are already documented elsewhere as the main predictors of IPIs. Low level of knowledge and practices in personal hygiene and environmental sanitation due to lack of access to education, low living standards of the community, and inadequate and unsafe water supply is often recognized as a major factor for the high prevalence of intestinal parasites among such communities. 
TABLE 4: Risk factors associated with Ascaris lumbricoides and hookworm infections.

\begin{tabular}{|c|c|c|c|c|c|}
\hline \multirow{2}{*}{ Risk factors } & \multicolumn{5}{|c|}{ Ascaris lumbricoides infection } \\
\hline & Positive no. (\%) & Negative no. (\%) & Total no. (\%) & $\operatorname{AOR}(95 \% \mathrm{CI})$ & $p$ value \\
\hline \multicolumn{6}{|l|}{ Age groups } \\
\hline Childhood & $4(50)$ & $4(50)$ & $8(2.1)$ & $2.621(0.631-19.039)$ & 0.341 \\
\hline Midchildhood & $65(20.3)$ & $255(79.7)$ & $320(83.5)$ & $0.686(0.317-1.405)$ & 0.287 \\
\hline Early adolescent & $18(32.7)$ & $37(67.3)$ & $55(14.4)$ & 1 & \\
\hline \multicolumn{6}{|l|}{ Residence } \\
\hline Rural & $46(25.3)$ & $136(74.7)$ & $182(47.5)$ & $0.96(0.545-1.715)$ & 0.908 \\
\hline Urban & $41(20.4)$ & $160(79.6)$ & $201(52.5)$ & 1 & \\
\hline \multicolumn{6}{|l|}{ Family size } \\
\hline $2-4$ & $12(26.7)$ & $33(73.3)$ & $45(11.7)$ & $2.706(1.043-7.017)$ & $0.041^{*}$ \\
\hline $5-7$ & $51(26.7)$ & $140(73.3)$ & $191(49.9)$ & $1.695(0.915-3.142)$ & \\
\hline $8-10$ & $24(16.3)$ & $123(83.7)$ & $147(38.4)$ & 1 & \\
\hline \multicolumn{6}{|l|}{ Family monthly income (ETB) } \\
\hline$<800$ & $57(35)$ & $106(65)$ & $163(42.6)$ & $2.004(0.709-5.667)$ & 0.190 \\
\hline $800-2,000$ & $23(13.5)$ & $147(86.5)$ & $170(44.4)$ & $1.035(0.356-3.011)$ & 0.950 \\
\hline$>2,000$ & $7(14)$ & $43(86)$ & $50(13.1)$ & 1 & \\
\hline \multicolumn{6}{|l|}{ Father education } \\
\hline Primary school & $60(27.1)$ & $161(72.9)$ & $221(57.7)$ & $1490(0.000)$ & 0.999 \\
\hline Secondary school and above & $27(16.7)$ & $135(83.3)$ & $162(40.5)$ & $1486(0.000)$ & 0.999 \\
\hline College and above & - & $7(100)$ & $7(1.8)$ & 1 & \\
\hline \multicolumn{6}{|l|}{ Father occupation } \\
\hline Daily laborer & $9(60)$ & $6(40)$ & $15(3.9)$ & $1.728(0.284-10.512)$ & 0.553 \\
\hline Farmer & $73(22)$ & $259(78)$ & $332(86.4)$ & $0.719(0.192-2.691)$ & 0.624 \\
\hline Govt. employee & $1(6.2)$ & $15(93.8)$ & $16(4.2)$ & $0.288(0.020-4.065)$ & 0.357 \\
\hline Merchant & $4(20)$ & $16(80)$ & $20(5.2)$ & 1 & \\
\hline \multicolumn{6}{|l|}{ Place of defecation } \\
\hline Toilet & $37(15.1)$ & $198(84.3)$ & $235(61.4)$ & 1 & \\
\hline Open field & $50(33.8)$ & $98(66.2)$ & $148(38.6)$ & $0.694(0.505-1.841)$ & 0.911 \\
\hline \multicolumn{6}{|l|}{ Drinking water sources } \\
\hline River & $49(32.9)$ & $100(67.1)$ & $149(38.9)$ & $1.675(0.915-3.142)$ & 0.112 \\
\hline Well & $9(33.3)$ & $18(66.7)$ & $27(7)$ & $1.937(0.704-5.332)$ & 0.201 \\
\hline Pipe & $29(14)$ & $178(86)$ & $207(54)$ & 1 & \\
\hline \multicolumn{6}{|c|}{ Fruit and vegetation washing habit before eating } \\
\hline Not at all & $7(38.9)$ & $11(61.1)$ & $18(4.7)$ & $1.422(0.410-4.929)$ & 0.579 \\
\hline Sometimes & $58(27)$ & $157(73)$ & $215(56.1)$ & $1.141(0.598-2.176)$ & 0.689 \\
\hline Always & $22(14.7)$ & $128(85.3)$ & $150(39.2)$ & 1 & \\
\hline \multicolumn{6}{|c|}{ Hand washing habit after toilet use } \\
\hline Not at all & $4(30.9)$ & $9(69.2)$ & $13(3.4)$ & $1.278(0.283-5.764)$ & 0.749 \\
\hline Sometimes & $60(29)$ & $147(71)$ & $207(54)$ & $1.428(0.756-2.698)$ & 0.273 \\
\hline Always & $23(14.1)$ & $140(85.9)$ & $163(42.6)$ & 1 & \\
\hline \multicolumn{6}{|l|}{ Ways of waste disposal } \\
\hline Open damp & $58(38.4)$ & $93(61.6)$ & $151(39.4)$ & $8.033(3.022-21.355)$ & $0.001^{* *}$ \\
\hline Burying & $23(25.3)$ & $68(74.7)$ & $91(23.8)$ & $4.898(1.722-13.929)$ & $0.003^{*}$ \\
\hline Burning & $6(4.3)$ & $135(95.7)$ & & 1 & \\
\hline \multicolumn{6}{|l|}{ Fingernail cleanliness } \\
\hline Not clean & $40(31.5)$ & $87(68.5)$ & $127(33.2)$ & $1.301(0.727-2.328)$ & 0.376 \\
\hline Clean & $47(18.4)$ & $209(81.6)$ & $256(66.8)$ & 1 & \\
\hline
\end{tabular}


TABLE 4: Continued.

\begin{tabular}{|c|c|c|c|c|c|}
\hline \multirow{2}{*}{ Risk factors } & \multicolumn{5}{|c|}{ Ascaris lumbricoides infection } \\
\hline & Positive no. (\%) & Negative no. (\%) & Total no. (\%) & AOR (95\% CI) & $p$ value \\
\hline \multicolumn{6}{|l|}{ Hookworm infection } \\
\hline \multicolumn{6}{|l|}{ Family size } \\
\hline $2-4$ & $5(11.1)$ & $40(88.9)$ & $45(11.7)$ & $0.513(0.169-1.555)$ & 0.238 \\
\hline $5-7$ & $43(22.6)$ & $148(77.5)$ & $191(49.9)$ & $1.112(0.619-1.998)$ & 0.722 \\
\hline $8-10$ & $31(21.1)$ & $116(78.9)$ & $147(38.4)$ & 1 & \\
\hline \multicolumn{6}{|c|}{ Family monthly income (ETB) } \\
\hline$<800$ & $41(25.2)$ & $122(74.8)$ & $163(42.6)$ & $1.038(0.375-2.873)$ & 0.943 \\
\hline $800-2,000$ & $31(18.2)$ & $139(81.8)$ & $170(44.4)$ & $1.314(0.484-3.562)$ & 0.592 \\
\hline$>2,000$ & $7(14)$ & $43(86)$ & $50(13.1)$ & 1 & \\
\hline \multicolumn{6}{|l|}{ Mother education } \\
\hline Primary school & $76(21.9)$ & $271(78.1)$ & $347(90.6)$ & $4893(0.000-000)$ & 0.999 \\
\hline Secondary school & $3(9.4)$ & $29(90.6)$ & $32(8.4)$ & $2958(0.000-000)$ & 0.999 \\
\hline College and above & - & $4(100)$ & $4(100)$ & 1 & \\
\hline \multicolumn{6}{|l|}{ Father education } \\
\hline Primary school & $54(24.4)$ & $167(75.6)$ & $221(57.7)$ & $0.208(0.021-2.027)$ & 0.176 \\
\hline Secondary school & $23(14.8)$ & $132(85.2)$ & $155(40.4)$ & $0.167(0.017-1.646)$ & 0.125 \\
\hline College and above & $2(28.6)$ & $5(71.4)$ & $7(1.8)$ & 1 & \\
\hline \multicolumn{6}{|l|}{ Mother occupation } \\
\hline Daily laborer & $1(33.3)$ & $2(66.7)$ & $3(0.8)$ & $2.015(0.103-39.620)$ & 0.645 \\
\hline Housewife & $76(21.7)$ & $274(78.3)$ & $350(9.4)$ & $1.685(0.331-8.576)$ & 0.530 \\
\hline Govt. employee & - & $9(100)$ & $9(2.3)$ & $0.000(0.000-0.000)$ & 0.999 \\
\hline Merchant & $2(9.5)$ & $19(90.5)$ & $21(5.57)$ & 1 & \\
\hline \multicolumn{6}{|l|}{ Place of defecation } \\
\hline Open field & $48(32.4)$ & $100(67.6)$ & $148(38.6)$ & $1.567(0.812-3.022)$ & 0.180 \\
\hline Toilet & $31(13.2)$ & $204(86.8)$ & $235(61.4)$ & 1 & \\
\hline \multicolumn{6}{|c|}{ Source of drinking water } \\
\hline River & $40(26.8)$ & $109(73.2)$ & $149(38.9)$ & $1.329(0.702-2.513)$ & 0.382 \\
\hline Well & $10(37)$ & $17(63)$ & $27(7)$ & $1.962(0.716-5.380)$ & 0.190 \\
\hline Pipe & $29(14)$ & $178(86)$ & $207(54)$ & 1 & \\
\hline \multicolumn{6}{|l|}{ Shoe-wearing habit } \\
\hline Not at all & $9(56.2)$ & $7(43.8)$ & $16(4.2)$ & $8.346(2.908-18.761)$ & $0.001^{* *}$ \\
\hline Sometimes & $35(36.8)$ & $60(63.2)$ & $95(24.8)$ & $3.648(2.094-6.358)$ & $0.001^{* *}$ \\
\hline Frequent & $35(12.9)$ & $237(87.1)$ & $272(71)$ & 1 & \\
\hline \multicolumn{6}{|c|}{ Hand washing habit after toilet use } \\
\hline Not at all & $5(38.5)$ & $8(61.5)$ & $13(3.4)$ & $1.547(0.408-5.867)$ & 0.521 \\
\hline Sometimes & $49(23.7)$ & $158(76.3)$ & $207(54)$ & $0.919(0.495-1.706)$ & 0.789 \\
\hline Always & $25(15.3)$ & $138(84.7)$ & $163(42.6)$ & 1 & \\
\hline \multicolumn{6}{|l|}{ Ways of waste disposal } \\
\hline Open damp & $36(23.8)$ & $115(76.2)$ & $151(39.4)$ & $1.686(0.761-3.736)$ & 0.198 \\
\hline Burying & $30(33)$ & $61(67)$ & $91(23.8)$ & $2.970(1.290-6.838)$ & $0.011^{*}$ \\
\hline Burning & $13(9.2)$ & $128(90.8)$ & $141(36.8)$ & 1 & \\
\hline \multicolumn{6}{|l|}{ Fingernail cleanliness } \\
\hline Not clean & $35(27.6)$ & $92(72.4)$ & $127(33.2)$ & $1.047(0.585-1.872)$ & 0.878 \\
\hline Clean & $44(17.2)$ & $212(82.8)$ & $256(66.8)$ & 1 & \\
\hline
\end{tabular}

Note: $1=$ reference value; ${ }^{* *}$ statistically significant at $p \leq 0.001 ;{ }^{*}$ statistically significant at $p<0.05, \mathrm{AOR}=$ adjusted odds ratio; multivariate regression model for age, residence, family size, family monthly income, father education, father occupation, place of defecation, source of drinking water, habit of eating unwashed fruits and vegetables, handwashing habit after toilet use, ways of waste disposal, and fingernails cleanliness for Ascaris lumbricoides infection, and residence, family size, family monthly income, father education, mother education, mother occupation, place of defecation, source of drinking water, handwashing habit after toilet use, ways of waste disposal, and fingernails cleanliness for hookworm infection. 
On the contrary, sex, age, family size, grade level, and raw meat-eating habits were not associated with IPIs. In many studies, males were found to be more exposed to IPIs than females $[23,24,37,44]$ due to differences in gender roles. In this study, however, male and female participants were found to be equally infected with intestinal parasites (Additional file 1). Previous studies in Jawi [25] and Tilili [36] towns (northwest Ethiopia) and Babile town [45] (Southern Ethiopia) have also shown similar observations. This may indicate that the differences in gender roles are narrowing. Regarding age, most of the participants (83.5\%) were within a similar age group (midchildhood), and as a result, the differences may not be expected within this narrow age range.

Regarding individual parasites identified, almost half of the participants were infected with parasitic helminths predominantly by Ascaris lumbricoides (close to 23\%) and Hookworms (about 21\%). A. lumbricoides infection is a worldwide problem mainly in tropical and subtropical countries where there are conducive conditions $[46,47]$. Such a high prevalence rate of $A$. lumbricoides among students in the first-cycle primary schools in Sasiga District may be attributed to one or more of the risk factors. The area is characterized mostly by moist, warm, and shaded soils which are suitable conditions for the parasite. Moreover, a significant proportion of the participants were from the rural area (47\%) and who dumped wastes in open area (39\%) both of which can be contributing factors as also shown in MVLRA (Table 4). A. lumbricoides was also found to be highly prevalent (11-23\%) among other schoolchildren in Ethiopia $[18,24,32]$. Hookworm infection has also been a global health problem and more prevalent in low-income countries [48-50]. Hookworm infection is also reported to be high (11-33\%) among schoolchildren in the different regions of Ethiopia [18, 21, 22, 25, 32, 37]. Walking barefoot in a warm climate, poor personal hygiene, and environmental sanitation are very important risk factors for hookworm infection. In this study, hookworm infection was significantly associated with shoe-wearing habits, ways of waste disposal, defecation habit, source of drinking water, fingernail cleanliness, and handwashing habit after toilet use (Tables 2 and 4 and Supplementary file 2).

Infection rates by the rest five parasites (identified in this study) were relatively low as compared to the reports from different parts of Ethiopia. However, S. mansoni infection cannot be underestimated due to its seriousness. About 17 (4.4\%) schoolchildren were found to be infected with $S$. mansoni, and the most important risk factor was age. Older children were more likely to get $S$. mansoni infection than younger ones (Supplementary file 3 ). In such communities, older children are more commonly engaged in field activities compared to youngsters that may expose them to S. mansoni infection.

\section{Conclusion}

Based on the result of this study, children attending in the first-cycle primary schools in Sasiga District are likely at high-risk of IPIs, showing that the burden of IPIs continues to be endemic in poor communities. A. lumbricoides and hookworm were the most prevalent intestinal parasites among the schoolchildren in the study area. Living in rural area, low family income, poor handwashing habit after toilet use, open-field defecation habit, lack of access to safe drinking water, inconsistence of shoe-wearing habit, inappropriate waste disposal methods, and unclean fingernails were found to be the most important predictors of IPIs among the students in the first-cycle primary school in Sasiga District. To reduce such a high burden of intestinal parasitic infections, effective strategies should be designed and implemented that involve decision makers, health workers, school teachers, the mass media, and community and religious leaders.

\section{Data Availability}

The [some statistical analyses and STROBE Statement] data used to support the findings of this study are included within the supplementary information file(s).

\section{Ethical Approval}

Before collecting the data, the ethical review committee of Science College, Bahir Dar University, cleared the study, and a letter describing the objective of the research was written to Sasiga District Administration Educational Office and the four Elementary Schools. Due to an issue of confidentiality and most of the children's parents/guardians could not understand written statements very well, verbal consent was obtained from the children's parents/guardians selected for the study after explaining the purpose and the procedures of the study. The study subjects who were positive for intestinal parasites were treated for free at the health centers or clinics they were diagnosed with specific drugs by a physician.

\section{Conflicts of Interest}

The authors declare that there is no conflict of interest regarding the publication of this article.

\section{Acknowledgments}

This research was funded by Bahir Dar University and the Ministry of Education, Ethiopia. These funding bodies do not have any roles in the design of the study and collection, analysis, and interpretation of data and in writing the manuscript. The authors forward special thanks to Bahir Dar University and the Ministry of Education for the financial support. The authors also like to thank health workers at health centers and clinics in Sasiga District, students, parents, and school community at the selected first-cycle primary schools.

\section{Supplementary Materials}

Supplementary 1. Supplementary material file 1: the association of IPIs with potential risk factors (sociodemographic, behavioral, personal hygienic practices, and environmental 
sanitation) among students in the first-cycle primary school in Sasiga District, southwest Ethiopia, 2018/2019.

Supplementary 2. Supplementary material file 2: the association of the risk factors related to personal, behavioral, and hygienic practices with the detected intestinal parasites among students in the first-cycle primary school in Sasiga District, southwest Ethiopia, 2018/2019.

Supplementary 3. Supplementary material file 3: multivariate logistic regression analysis of potential risk factors associated with Entameoba histolytica/dipar, Trichuris trichiura, Giardia intestinalis, Hymenolepis nana, and Schistosoma mansoni infections among school children in Sasiga District, southwest Ethiopia, 2019.

Supplementary 4. Supplementary material file 4: STROBE Statement-Checklist of items that are included in the reports of this cross-sectional studies on the prevalence of IPIs among students in the first-cycle primary school in Sasiga District, southwest Ethiopia, 2018/2019. 22 STROBE checklists for cross-sectional studies were included here.

\section{References}

[1] WHO, Working to overcome the global impact of neglected tropical diseases: first WHO report on neglected tropical diseases, WHO, Geneva, 2010.

[2] P. Collier, The Bottom Billion: Why the Poorest Countries Are Failing and What Can Be Done about it Oxford, Oxford University Press, 2007.

[3] P. J. Hotez, A. Fenwick, L. Savioli, and D. H. Molyneux, "Rescuing the bottom billion through control of neglected tropical diseases," The Lancet, vol. 373, no. 9674, pp. 1570-1575, 2009.

[4] P. J. Hotez, M. Alvarado, M. G. Basáñez et al., "The global burden of disease study 2010: interpretation and implications for the neglected tropical diseases," PLoS Neglected Tropical Diseases, vol. 8, no. 7, pp. 203-308, 2014.

[5] S. C. Ojha, C. Jaide, N. Jinawath, P. Rotjanapan, and P. Baral, "Geohelminths: public health significance," Journal of Infection in Developing Countries, vol. 8, no. 1, pp. 5-16, 2014.

[6] F. Samuel, "Status of soil-transmitted helminths infection in Ethiopia," American Journal of Health Research, vol. 3, no. 3, pp. 170-176, 2015.

[7] A. Efstratiou, J. E. Ongerth, and P. Karanis, "Waterborne transmission of protozoan parasites: review of worldwide outbreaks-an update 2011-2016," Water Research, vol. 114, pp. 14-22, 2017.

[8] S. Brooker, "Estimating the global distribution and disease burden of intestinal nematode infections: Adding up the numbers - A review," International Journal for Parasitology, vol. 40, no. 10, pp. 1137-1144, 2010.

[9] A. Alum, J. R. Rubino, and M. K. Ijaz, "The global war against intestinal parasites-should we use a holistic approach?," International Journal of Infectious Diseases, vol. 14, no. 9, pp. e732e738, 2010.

[10] S. M. Davis, J. M. Montgomery, K. O. Odero et al., "Soil-transmitted helminths in pre-school-aged and school-aged children in an urban slum: a cross-sectional study of prevalence, distribution, and associated exposures," The American Journal of
Tropical Medicine and Hygiene, vol. 91, no. 5, pp. 1002-1010, 2014.

[11] A. H. A. de Moraes Neto, A. P. M. F. Pereira, M. d. F. L. Alencar et al., "Prevalence of intestinal parasites versus knowledge, attitudes, and practices of inhabitants of lowincome communities of Campos dos Goytacazes, Rio de Janeiro State, Brazil," Parasitology Research, vol. 107, no. 2, pp. 295-307, 2010.

[12] N. Padmaja, S. P. Swaroop, and P. Nageswararao, "Prevalence of intestinal parasitic infections among schoolchildren in and around Amalapuram, India," Journal of Health and Medical Research, vol. 2, no. 2, pp. 36-44, 2014.

[13] S. Erismann, S. Diagbouga, P. Odermatt et al., "Prevalence of intestinal parasitic infections and associated risk factors among schoolchildren in the Plateau Central and CentreOuest regions of Burkina Faso," Parasites \& Vectors, vol. 9, no. 1, p. 554, 2016.

[14] G. A. Alwabr and E. E. AL-Moyed, "Prevalence of intestinal parasitic infections among schoolchildren of AL-Mahweet Governorate Yemen," European Journal of Biological Research, vol. 6, no. 2, pp. 64-73, 2016.

[15] M. B. Ajayi, A. H. Sani, E. SMC, E. E. Afocha, and A. A. Adesesan, "Intestinal parasitic infection and body mass index among school children in Oshodi lagos Nigeria," Advances in Cytology \& Pathology, vol. 2, no. 2, pp. 44-49, 2017.

[16] B. Choi and B. Kim, "Prevalence and risk factors of intestinal parasite infection among schoolchildren in the peripheral highland regions of Huanuco, Peru," Osong Public Health and Research Perspectives, vol. 8, no. 5, pp. 302-307, 2017.

[17] U. Living-Jamala, N. C. Eze, and F. O. Nduka, "Prevalence and intensity of intestinal helminth infections and associated risk factors among school-aged children in Abua/Odual local government area, Rivers state," Journal of Applied Life Sciences International, vol. 16, no. 2, pp. 1-7, 2018.

[18] A. Abossie and M. Sied, "Assessments of the prevalence of intestinal parasitic infection and associated risk factors among primary schoolchildren in Chencha town southern Ethiopia," BMC Public Health, vol. 14, no. 166, pp. 47-58, 2014.

[19] B. Mathewos, A. Alemu, D. Woldeyohannes et al., "Current status of soil transmitted helminths and Schistosoma mansoni infection among children in two primary school in north Gonder north-west Ethiopian a cross-sectional study," $B M C$ Research Notes, vol. 7, no. 1, p. 88, 2014.

[20] T. Workneh, E. Esmael, and M. Ayichiluhm, "Prevalence of intestinal parasitic infections and associated factors among Debre Elias primary school, East Gojjam Zone Amhara Region, north-West Ethiopia," Journal of Bacteriology \& Parasitology, vol. 5, no. 181, pp. 11-19, 2014.

[21] A. Ayalew, T. Debebe, and A. Worku, "Prevalence and risk factors of intestinal parasites among Delgi schoolchildren, North Gondar, Ethiopia," Journal of Parasitology and Vector Biology, vol. 3, no. 5, pp. 75-81, 2011.

[22] M. Andualem, "Parasitic infection and associated factors among the primary school children in Motta town, Western Amhara, Ethiopia," American Journal of Pharmacy and Health Research, vol. 2, no. 6, pp. 248-254, 2014.

[23] G. Gebretsadik, "Prevalence of intestinal Parasites and associated risk factors among schoolchildren of Homesha District (Woreda) in Benishangul-Gumuz regional State, western Ethiopia," Journal of Family Medicine and Health Care, vol. 2, no. 4, pp. 57-64, 2016. 
[24] T. Hailegebriel, "Prevalence of intestinal parasitic infections and associated risk factors among students at Dona Berber Primary school, Bahir Dar, Ethiopia," BMC Infectious Diseases, vol. 17, no. 1, p. 362, 2017.

[25] B. Sitotaw, H. Mekuriaw, and D. Damtie, "Prevalence of intestinal parasitic infections and associated risk factors among Jawi primary school children, Jawi town, north-west Ethiopia," BMC Infectious Diseases, vol. 19, no. 1, p. 341, 2019.

[26] Y. Yimam, A. Degarege, and B. Erko, "Effect of anthelminthic treatment on helminth infection and related anaemia among school-age children in north-western Ethiopia," BMC Infectious Diseases, vol. 16, no. 1, p. 613, 2016.

[27] M.-E. Sackev, "Intestinal factors and parasite infections: prevalence, risk factors and consequences for child growth, iron status and development in rural Ecuador. Msc. Thesis," Virginia Polytechnic and State University, Ecuador, 2001.

[28] A. A. M. Ahmed, A. A. Afifi, E. M. Malik, and I. Adam, "Intestinal protozoa and intestinal helminthic infections among schoolchildren in Central Sudan," Asian Pacific Journal of Tropical Medicine, vol. 3, no. 4, pp. 292-293, 2010.

[29] N. Emile, N. Bosco, and B. Karine, "Prevalence of intestinal parasitic infections and associated risk factors among Kigali Institute of Education students in Kigali. Rwanda," Tropical Biomedicine, vol. 30, no. 4, pp. 718-726, 2013.

[30] M. Legesse and B. Erko, "Prevalence of intestinal parasites among school children in a rural area close to the southeast of Lake Langano, Ethiopia," Ethiopian Journal of Health Development, vol. 18, no. 2, pp. 117-120, 2005.

[31] G. Bugssa, B. Dessalegn, M. Alemu, H. Desta, and T. Kahsay, "A survey of intestinal parasitic infections among Dega Ochollo primary School Children, Ochollo, South Ethiopia," Science Journal of Public Health, vol. 3, no. 1, pp. 56-60, 2015.

[32] M. Alamir, W. Awoke, and A. Feleke, "Intestinal parasites infection and associated factors among schoolchildren in Dagi primary school Amhara National Regional State, Ethiopia," Health, vol. 5, no. 10, pp. 1697-1701, 2013.

[33] WHO, Basic laboratory method in Medical Parasitology., WHO, Geneva, 1991.

[34] S. Lemeshow, R. X. Sturdivant, and D. W. Hosmer, "Applied logistic regression," Wiley series in probability and statistics, 2013.

[35] A. Abera and E. Nibret, "Prevalence of gastrointestinal helminths infection and associated risk factors among schoolchildren in Tilili town north-west," Asian Pacific Journal of Tropical Disease, vol. 2, no. 14, pp. 525-530, 2014.

[36] T. Alelign, A. Degarege, and B. Erko, "Prevalence and factors associated with undernutrition and anaemia among school children in Durbete town, northwest Ethiopia," Archives of Public Health, vol. 73, no. 1, pp. 34-38, 2015.

[37] M. Abdi, E. Nibret, and A. Munshea, "Prevalence of intestinal helminthic infections and malnutrition among schoolchildren of the Zegie peninsula, northwestern Ethiopia," Journal of Infection and Public Health, vol. 10, pp. 84-92, 2017.

[38] R. Sehgal, G. V. Reddy, J. J. Verweij, and A. V. Rao, "Prevalence of intestinal parasitic infections among schoolchildren and pregnant women in a low socio-economic area, Chandigarh, North India," Reviews in Infection, vol. 1, no. 2, pp. 100-103, 2010.

[39] S. P. Sherchand, D. R. Joshi, N. Adhikarr et al., "Intestinal parasitosis among school going children, Nepal," Journal of Health and Allied Sciences, vol. 1, no. 1, pp. 12-15, 2010.
[40] K. Yadav and S. Prakash, "Study of intestinal Parasitosis among school children of Kathmandu Valley, Nepal," Asian Journal of Biomedical and Pharmaceutical Sciences, vol. 6, no. 59 , p. 40, 2016.

[41] O. M. Agbolade, N. C. Agu, O. O. Adesanya et al., "Intestinal helminthiases and schistosomiasis among school children in an urban center and some rural communities in southwest Nigeria," The Korean Journal of Parasitology, vol. 45, no. 3, pp. 233-238, 2007.

[42] J. G. Damen, J. Luka, E. I. Biwan, and M. Lugos, "Prevalence of intestinal parasites among pupils in rural north Eastern, Nigeria," Nigerian Medical Journal, vol. 52, no. 1, pp. 4-6, 2011.

[43] I. Sangaré, S. Bamba, M. Cissé et al., "Prevalence of intestinal opportunistic parasites infections in the university hospital of Bobo-Dioulasso, Burkina Faso," Infectious Diseases of Poverty, vol. 4, no. 1, p. 32, 2015.

[44] E. O. Dada, "Prevalence of human intestinal helminths among primary schoolchildren in Ipogun, Ifedore local government area Nigeria," Journal of Global Biosciences, vol. 5, no. 1, pp. 3401-3407, 2016.

[45] G. Tadesse, "Prevalence of intestinal helminthic infection and associated risk factors among schoolchildren in Babile town eastern Ethiopia," Ethiopian Journal of Health Sciences, vol. 19, no. 2, pp. 49-51, 2005.

[46] M. O. Harhay, J. Horton, and P. L. Olliaro, "Epidemiology and control of human gastrointestinal parasites in children," Expert Review of Anti-infective Therapy, vol. 8, no. 2, pp. 219-234, 2010.

[47] C. Dold and C. V. Holland, “_Ascaris_ and ascariasis," Microbes and Infection, vol. 13, no. 7, pp. 632-637, 2011.

[48] A. Fenwick, "The global burden of neglected tropical diseases," Public Health, vol. 126, no. 3, pp. 233-236, 2012.

[49] P. J. Hotez, J. Bethony, M. E. Bottazzi, S. Brooker, and P. Buss, "Hookworm: the great infection of mankind," PLoS Medicine, vol. 2, no. 3, article e67, 2005.

[50] J. Bethony, S. Brooker, M. Albonico et al., "Soil-transmitted helminth infections: ascariasis, trichuriasis, and hookworm," The Lancet, vol. 367, no. 9521, pp. 1521-1532, 2006. 